\title{
Design-of-Experiments Analysis of Li-Ion Cell Capacity Fading in High Temperature Automotive Conditions
}

\author{
Richard Stocker \\ Horizon Scanning \\ HORIBA MIRA \\ Nuneaton, UK \\ Richard.Stocker@Horiba-MIRA.com
}

\author{
Asim Mumtaz \\ Stephenson Institute for Renewable \\ Energy \\ University of Liverpool \\ Liverpool, UK \\ A.Mumtaz@liverpool.ac.uk
}

\author{
Neophytos Lophitis \\ Institute for Future Transport and Cities \\ Coventry University \\ Coventry, UK \\ N.Lophitis@ coventry.ac.uk
}

\begin{abstract}
This paper examines the evolution of performance degradation through capacity fade in $\mathrm{Li}$-ion cells when subjected to 8 months of automotive drive cycles in high temperature conditions. This is done by combining a temperature controlled, highly transient cycling approach with a design-of-experiments matrix varying charge current magnitude and Depth-of-Discharge, two factors known to be influential in ageing rate. The subsequent results are then analyzed using design-of-experiments methodology and trend analysis, comparing the rates of capacity evolution at various points in cell lifetime. What was found is that the cell ageing evolved in 3 main phases dependent on the cycling time duration rather than Ah throughput. In early life, charge current was influential, while in later life depth-of-discharge proved significant. It was also clear, that the factors could not be treated in isolation, with higher order and interaction effects observed. This implies that modeling of cell ageing cannot be uniform across lifetime and must consider the different ageing phases of the cell.
\end{abstract}

Keywords - Lithium-ion, battery cell, simulation, model, equivalent circuit

\section{INTRODUCTION}

This paper looks at high temperature Li-ion cell ageing in automotive conditions, evaluating how usage conditions affect capacity degradation. Vehicle electrification is a key contemporary issue due to the widespread aim to reduce automotive emissions and limit the use of the internal combustion engine (ICE) [1], [2].

One of the biggest concerns with Li-Ion battery packs used in automotive cases is longevity, with vehicles requiring at least a 5 year lifetime [3], [4] to maintain at least $80 \%$ of original cell capacity [5].

To achieve this, battery cell ageing must be well understood, to create effective battery models and management/control strategies for estimating and mitigating resulting behavior changes.
This is of particular importance because Li-ion cell ageing is very complex [6], [7], and dependent on usage conditions such as temperature, State-of-Charge (SoC) and applied current [8], [9]. There is also added complexity due to battery cells being effectively black-box, with only full cell voltage measurements such as full cell current, voltage and temperature being visible, while it has been shown that ageing mechanisms can develop on individual components within the cell [10] and heterogeneously within the cell geometry [11], [12].

With the complex nature of cell ageing, Design-ofexperiments (DoE) approaches have been used to identify the most significant contributions [13], [14]. It has also been shown that ageing depends significantly, on the profile used to cycle the cells [15], [16].

This paper shows a combination of the above to give a testing approach designed to represent automotive conditions combined with a design-of-experiments approach of the key parameters that can be varied through Battery Management System (BMS) control. This paper focuses on the capacity evolution, with resistance behavior investigated in a separate publication.

\section{METHODOLOGY}

\section{A. Cell Properties}

The cells used were 28Ah PHEV2 format NickelManganese-Cobalt (NMC)/graphite Li-Ion cells, designed for power orientated automotive applications.

\section{B. Cell Ageing Testing Approach}

The priority for the testing strategy was to make the testing conditions tightly controlled, make the results representative of automotive conditions, and create a significant amount of ageing to analyse through cell lifetime. It is known that high temperatures significantly accelerate Li-Ion cell ageing [17]. 
Therefore, to get significant ageing rates, the tests were ran at $45^{\circ} \mathrm{C}$, as that forms the upper end of automotive conditions, without activating the changes in nature of ageing that occur at very high temperatures [6], [7], [18]. In addition to this, there are distinct ageing mechanisms that occur within a cell based on operating temperature [13], [14] with high temperatures likely to show domination of only a few mechanisms, easing analysis. To ensure that the temperature conditions were tightly controlled during the cycling, ambient and active temperature control was used. The cooling plate setup is shown in Figure 1.

It has been shown,that the current profile used to cycle a battery cell influences how the cell ages, in addition to the magnitude of the currents being used [15], [19]. It can also indirectly affect ageing through internal heat generation and distribution effects [20], which influence ageing indirectly. The drive cycle chosen was the Federal Urban Driving Schedule (FUDS), as specified in [21], due to its basis on automotive demands. The cells were discharge cycled with this profile, then Constant Current Constant Voltage (CC$\mathrm{CV}$ ) charged at the test charge C-Rate. This was repeated through the test Depth-of-Discharge condition for 8 months or until the cell could no longer by cycled. Cell 1 failed after 7 months while all others lasted the full duration.

Reference performance tests were performed before the testing start and at 1 month intervals throughout the testing. These were performed at $25^{\circ} \mathrm{C}$, and consisted of $\mathrm{C} / 3$ and $1 \mathrm{C}$ constant current cycles and Hybrid Pulse Power Characterization (HPPC) tests which were cycled across the full usable range of the cell. These were used to supply more comprehensive data into the cell characteristics at each stage of ageing.

\section{Design of Experiments Approach}

Li-ion cell ageing is influenced by several factors [13], [14], [18], [22] - [24] and strong interaction effects between those factors, making test strategy construction complex. It has been shown that of particular importance is charge current magnitude [14], [25] and Depth-of-Discharge (DoD) range [13], [14], [19], [26]. Based on this, these two parameters form the basis of the experimental matrix. Two other factors identified as important are temperature [27], [28] and average SoC [7], [29]. To keep the change in cell degradation a function of the two purposely varied factors, average SoC was kept the same for all test cases and temperature was both kept the same, and actively controlled to account for the different levels of heat generation from varying charge current, as described in section II.B.

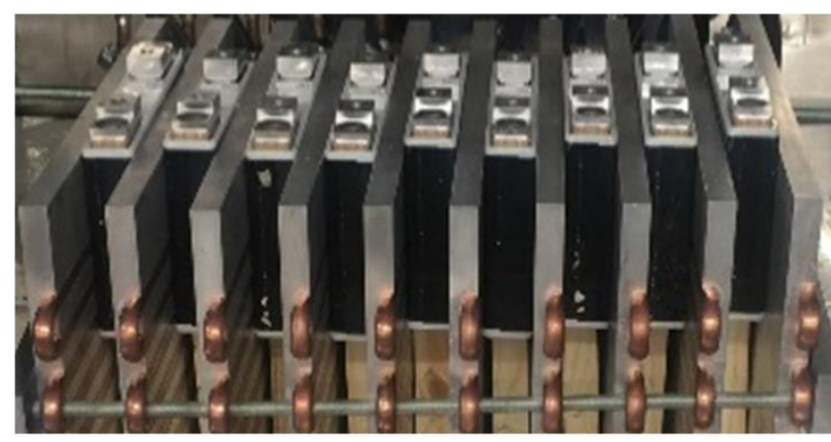

Fig. 1. Cell Testing Setup
TABLE I. TEST CASE DeFinition For CELl TESTS

\begin{tabular}{|c|c|c|c|c|c|c|c|c|c|}
\hline & \multicolumn{7}{|c|}{ Cell Test Cases } \\
\hline $\begin{array}{c}\text { Test } \\
\text { Case }\end{array}$ & 1 & 2 & 3 & 4 & 5 & 6 & 7 & 8 & 9 \\
\hline $\begin{array}{c}\text { SoC } \\
\text { Range }\end{array}$ & $15-85$ & $10-90$ & $5-95$ & $15-85$ & $10-90$ & $5-95$ & $15-85$ & $10-90$ & $5-95$ \\
\hline $\begin{array}{c}\text { Charge } \\
\text { C-Rate }\end{array}$ & 0.5 & 0.5 & 0.5 & 1 & 1 & 1 & 2 & 2 & 2 \\
\hline
\end{tabular}

It has been shown that SoC range and charge current do have a strong interaction effect [22] and due to the nonlinear behavior of cell ageing, are likely to be higher order. Due to these considerations, a full factorial approach was taken, with 3 levels for charge current and for SoC, which created 9 test cases. The labeling of each test case, and the associated parameters, are shown in Table 1.

Ageing is not a function just of usage conditions, but it is also dependent on usage history itself. For this reason, the ageing profiles for each cell were examined, and based on this different section of the ageing were analysed independently based on their perceived stage of ageing. To analyse each stage fairly between cells, the rate of capacity change with Ah throughput and time was measured, being scaled as a fraction of the original capacity of each cell. To get the rate of capacity change with each input metric (time and Ah throughput), the nature of the capacity change in each stage was assumed to be linear. This is shown to be a fair representation by Figure 2 which shows a relative capacity estimation error of less than $0.5 \%$ when comparing stage 2 trendline estimation to real datapoints when using both Ah throughput and time as inputs.

The capacity used for reference in each case was taken from the net capacity throughput of the HPPC test. The HPPC test conditions started after, and ended with, a C/50 CV stage, making the results representative of true capacity.

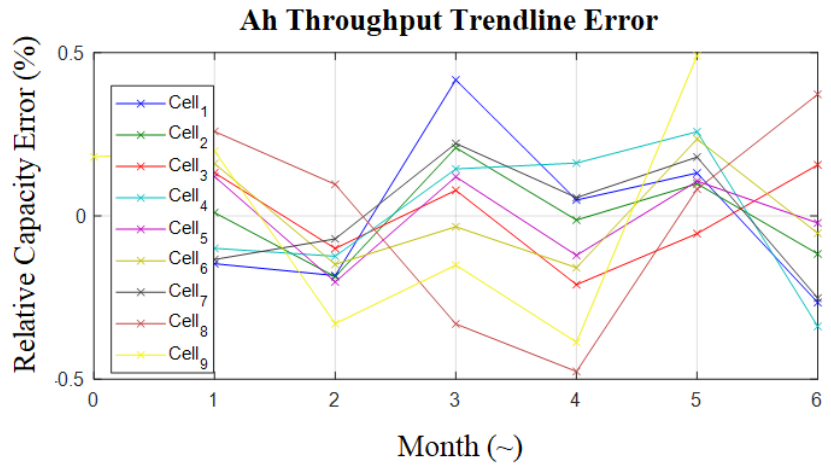

Cycle Time Trendline Error

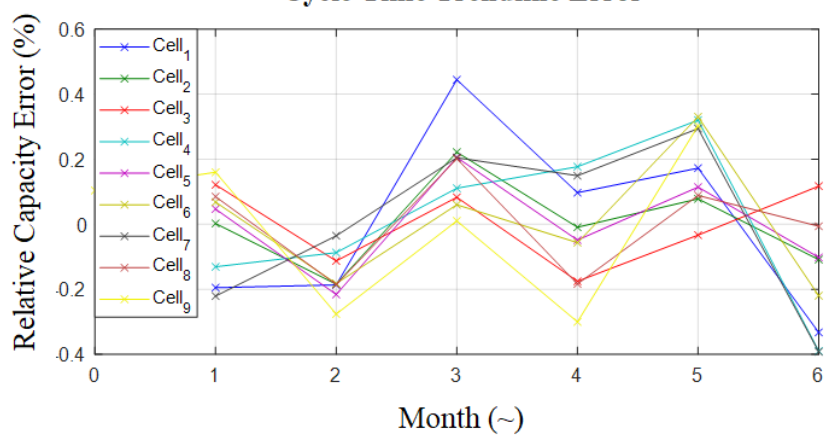

Fig. 2. Error with month when using linear trends to estimate capacity, relative to actual datapoints 
When possible, i.e. when all cells observed the same ageing stage, the variation of each factor was analyzed using mean factor analysis. For this, the variation in each factor was expressed by comparing the average value of each factor level when considering all test results with that level, normalised to the average of the entire test matrix.

The mean factor analysis estimates variation as a result of individual factor variation but does not highlight interaction effects. To analyse this, a response model, of the format shown in (1), was used to examine the relative importance of higher order and interaction effects, to give a full picture of the relationship between the DoE parameters and the rate of ageing. A corresponds to charge current magnitude, and $\mathrm{B}$ corresponds to DoD level, with $\mathrm{Y}$ being rate of capacity decrease.

$Y=\theta_{1}+\theta_{2} A+\theta_{3} B+\theta_{4} A B+\theta_{5} A^{2}+\theta_{6} B^{2}+\theta_{7} A^{2} B+\theta_{8} A B^{2}+\theta_{9} A^{2} B^{2}$

To do this analysis, each factor was assigned levels as shown in Table 2. Part of this study is evaluating the relative impacts of capacity throughput and time, so a response model was created for each input.

TABLE II. ASSIGNMENT OF LEVELS TO DoE FACTORS

\begin{tabular}{|c|c|c|c|}
\hline & \multicolumn{3}{|c|}{ Range of Levels } \\
\hline Level & -1 & 0 & 1 \\
\hline Charge C-Rate & 0.5 & 1 & 2 \\
\hline SoC Range & $15-85$ & $10-90$ & $5-95$ \\
\hline
\end{tabular}

\section{RESULTS}

\section{A. Capacity Fade Stages}

When analyzing Li-Ion capacity fading, the behavior can be nonlinear not just as a function of usage condition and cell design, but also as a function of usage itself (capacity throughput and cycle time). To apply DoE analysis appropriately, it should be applied to regions of cell ageing that are consistent.

To investigate the regions where this is valid, the relative cell capacity evolution is expressed for all cells as a function of cycle time and capacity throughput in Figure 3 and Figure 4 respectively. From both plots, it can be seen that ageing does not follow a consistent trend throughout the test duration. There are three main phases that are visible in the test data. The first is a high rate of capacity fade in the first month.

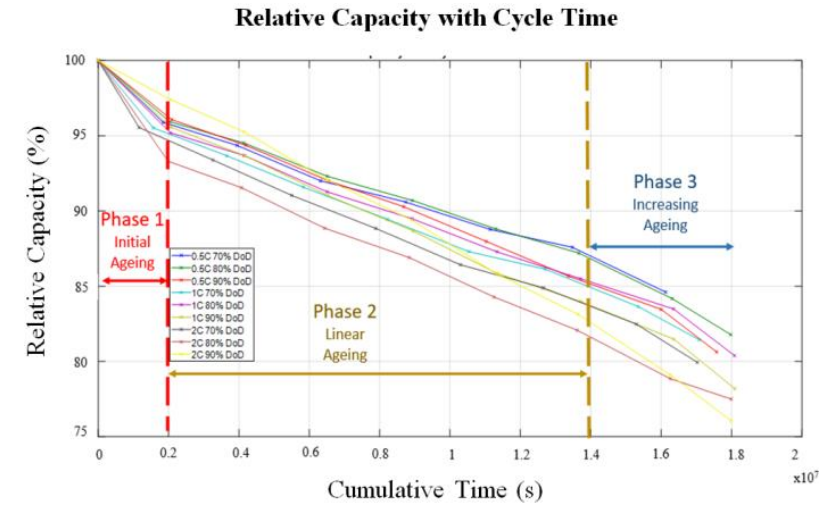

Fig. 3. Relative capacity throughput with cumulative time for all test conditions

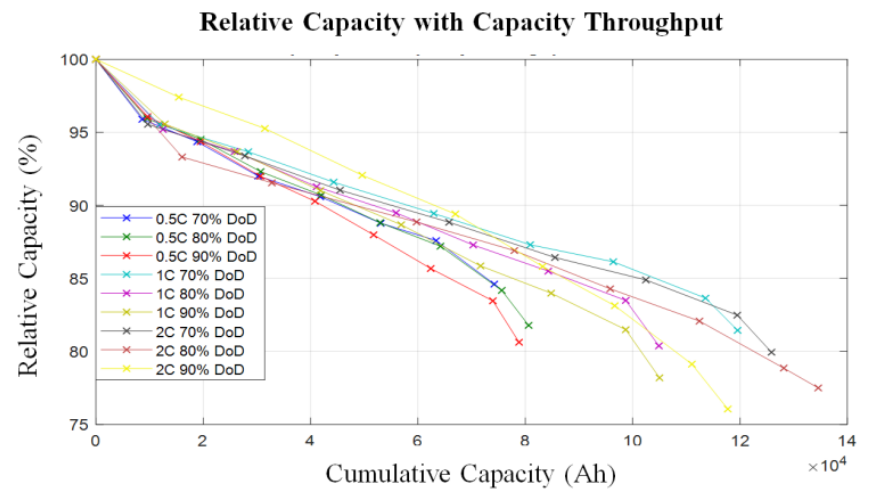

Fig. 4. Relative capacity throughput with cumulative capacity for all test conditions

The second is a relatively slow and linear fade across most of the test duration, and the last is the appearance of possible acceleration in the rate of ageing of the cell, which depending on the cell sample appears in the last 1-2 months of cell lifetime.

What can be concluded from this, is that the relationship between capacity fade and usage input (cycle time or Ah throughput) does change at different phases in the cell lifetime, but the largest section of ageing (Months 1-6) appears to be approximately linear. This is highlighted in Figure 5. In the first month, the rate of change is very high, with a steady capacity fade after that. In late months the rate increases, with the exact position cell dependent.

When observing Figure 4, it is hard to see a consistent Ah throughput value that causes the transition between the phases, particularly the late shift to accelerated ageing. When observing Figure 3, the shape of the profiles with respect to time is similar. This suggests that time spent at the $45^{\circ} \mathrm{C}$ test temperature is the dominant factor in the type of ageing a cell is observing. From Figure 4 it can also be seen that the ageing accelerates at between $82-85 \% \mathrm{SoH}$ for most cells, suggesting the capacity fade itself could also be a trigger.

It can also be observed that there are two anomalies to the observed trends. Cell 9 (2C charge rate and 90\% DoD) does not see the same initial capacity fade as the other cells, being linear from the start of testing. This suggests it may have begun life in an already more aged state, possibly due to more mature Solid Electrolyte Interphase (SEI) layer formation on the negative electrode.

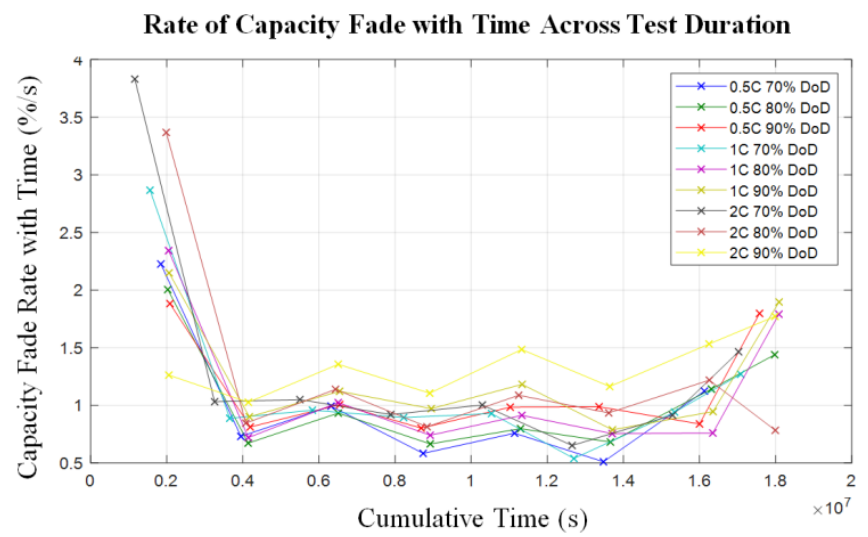

Fig. 5. Rate of Capacity Fade relative to time with Cumulative time for all cells 
Cell 8 does also not show the same sharp decrease in capacity towards the end-of-life that the other cells see. This is surprising as it is subjected to an ageing condition expected suffer a higher rate of degradation. This reflects the strong influence of cell variation in ageing that can be present in test results. Cell 1 also failed unexpectedly during Month 8 of cycling, so cannot be evaluated at the last datapoint.

\section{B. Influence of Charge Current and Depth-of-Discharge}

As it has been shown the profile changes into 3 distinct phases, it is likely that in each of these phases different ageing behavior is happening. For this reason, the phases will be examined separately. For all phases the variance in relative capacity fade rate with Ah throughput and time is shown. As all cells go through phase 2, additional analysis can be performed via mean factor analysis and response model creation.

\section{1) Phase 1}

Capacity Fade with Ah throughput and cycle time is shown in Figure 6. In phase 1 it is quite clear, that capacity fade correlates significantly with Charge C-Rate when being compared to cycle time more than the other phases. This suggests that in this phase, cycling the cell increases ageing, likely due to speeding up the layer formation on the negative electrode either directly, or indirectly through internal heating. The influence of charge current appears to increase significantly between $1 \mathrm{C}$ and $2 \mathrm{C}$ relative to between $0.5 \mathrm{C}$ and $1 \mathrm{C}$, suggesting a threshold in this phase that causes accelerated ageing. In this phase, Depth-of-Discharge is relatively insignificant, with the results showing a slight trend towards lower DoD increasing ageing rate. The relationships between usage factors and cycle time, appear to be more consistent than those using Ah throughput.

Relative Capacity Fade with Capacity Throughput Phase 1

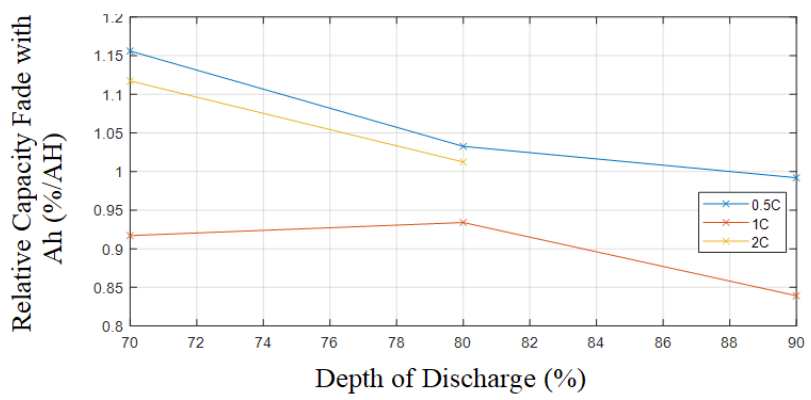

Relative Capacity Fade with Cycle Time Phase 1

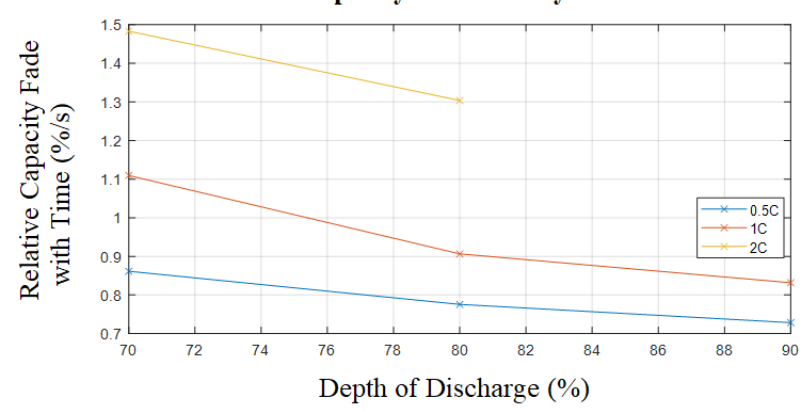

Fig. 6. Relative capacity fade rate with test condition for ageing phase 1 relative to a) $\mathrm{Ah}$ and b) time
Relative Capacity Fade with Capacity Throughput Phase 2

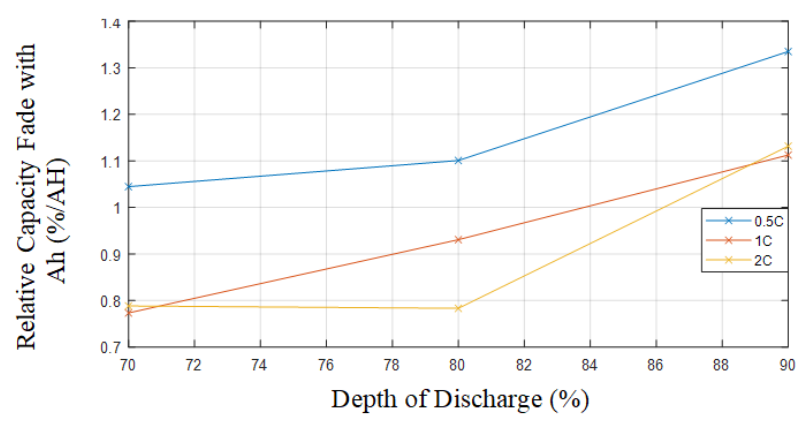

Relative Capacity Fade with Cycle Time Phase 2

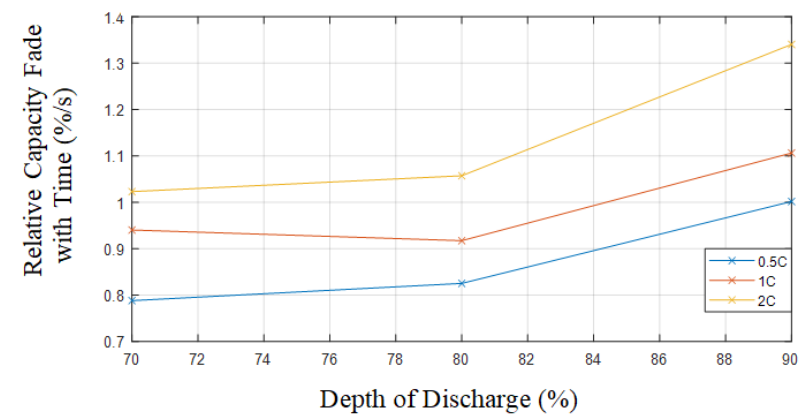

Fig. 7. Relative capacity fade rate with test condition for ageing phase 2 relative to a) Ah and b) time

\section{2) Phase 2}

Figure 7 shows rate of capacity fade for each test condition, relative to Ah throughput and time. In addition, the mean factor analysis for charge C-Rate and DoD changes are shown in Figure 8, scaling the results relative to the overall average for all test conditions. It can be seen that the variance of usage factors is significant, with the worstcase condition causing degradation at approximately 1.6 times the best-case condition for both time and capacity.

In all cases, increased DoD accelerates ageing. In most cases however, it appears that the relationship between DoD and ageing is not linear. Between $70-80 \%$ there is a relatively small increase in ageing, but between $80-90 \%$ it is much more significant, when comparing to both time and capacity throughput, suggesting the relationship is complex.

For charge C-Rate, the effect on ageing depends on the comparison input being time or capacity throughput. When comparing to cycle time, a higher charge C-Rate increases ageing in an approximately linear way. When comparing to capacity throughput however, a lower charge current magnitude is more degrading. This is due to lower charge currents effectively increasing time per cycle. With time being the apparent dominant influence on ageing, the longer cycle times dominate any additional ageing that occurs from higher charge currents. What can be seen however, is that the rate of change from $0.5 \mathrm{C}$ to $1 \mathrm{C}$ is significantly higher than $1 \mathrm{C}$ to $2 \mathrm{C}$, which indicates that charge current itself may become more significant itself above $1 \mathrm{C}$. When comparing to time, there is an approximately linear increase in capacity fade with charge C-Rate, indicating either that increased Ah throughput has an effect, or that increasing charge rate itself is causing accelerated ageing. 
Another observation from Figure 7 is that there is likely some interaction effects between Depth-of-Discharge and charge C-Rate.

This is indicated by the increase in degradation for with DoD being more pronounced as charge C-Rate increases, particularly at higher DoD. It is unclear visually whether the interaction is significant or due to natural testing variation.

3) Response Equations for time and Ah throughput Phase 2

As highlighted in Section III.B.2), the influence of each usage parameter (Charge C-Rate and DoD) is observed to be higher order. In addition, there is also a possibility of interaction effects between the two parameters. This limits the use of the mean-factor analysis and necessitates the use of a response model to identify the relative importance of higher order and interactive effects. The results for the response models for time and Ah throughput are shown in Figure 9, with a corresponding to charge C-Rate and B corresponding to DoD. What can be seen, is that for Ah throughput, the low-level factors dominate, with Crate and DoD having a strong decreasing and increasing effect on capacity fade rate respectively.
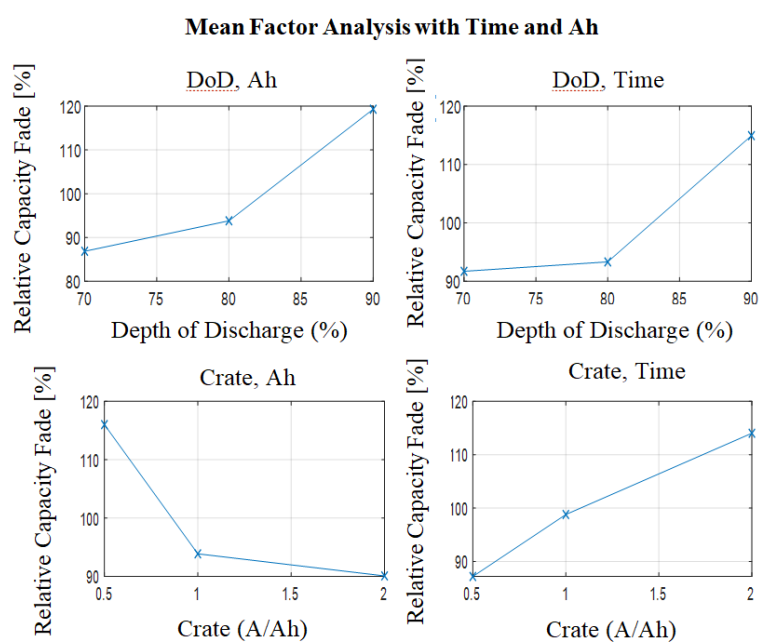

Fig. 8. Mean factor analysis with time and Ah throughput during phase 2

Response Model Coefficients for Capacity Fade Rate
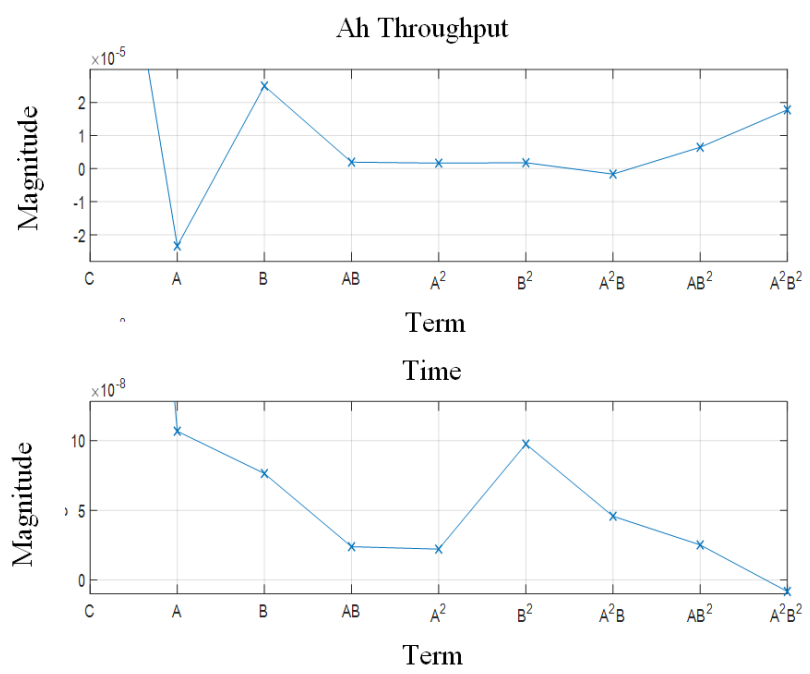

Fig. 9. Response Model Coefficients for a) time and b) Ah

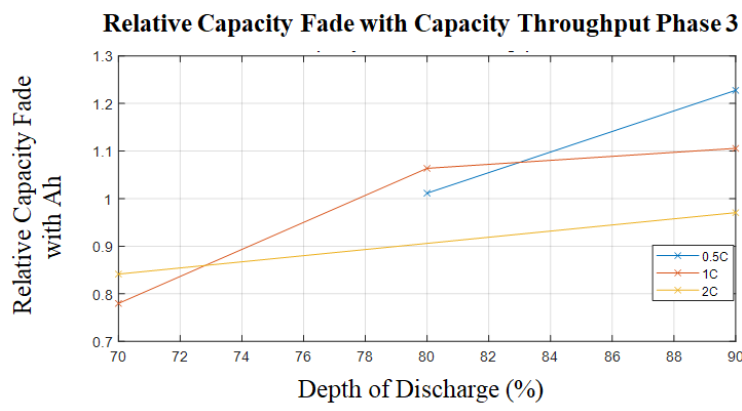

Relative Capacity Fade with Cycle Time Phase 3

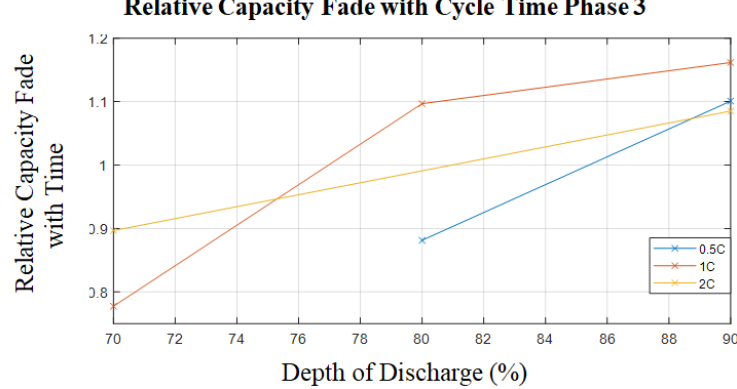

Fig. 10. Relative capacity fade rate with test condition for ageing phase 3 relative to a) Ah and b) time

The other significant affects are the higher order interaction effects. This does not seem physically realistic however. The time based trends are more consistent with expectations. Again, the first order effects are significant for charge C-Rate and DoD increase (this time both increasing degradation), while higher order DoD is also significant (matching with the trends observed in Figure 6. It can be seen however, that except for the highest order interaction effect, all interaction and higher order effects contribute significantly, confirming that the ageing is complex and that linear and isolated analysis of the ageing trends is not sufficient.

\section{4) Phase 3}

In this phase, there appears to be a strong correlation between degradation rate and DoD relative to both time and Ah throughput as shown in Figure 10. This suggests that eventually, higher Depth-of-Discharge conditions do lead to more significant ageing, although the ageing mechanisms they cause may not act straight away. Charge current is less conclusive. When compared to time it is suggested that medium charge currents cause higher levels of ageing, although there is much cross over between currents which does not make the analysis certain. In this case there is not a sufficient amount of datapoints to create a response model to analyse the influence of effects fully.

\section{CONCLUSIONS}

The analysis has revealed a significant amount about the ageing behavior of an NMC/graphite cell in high temperature automotive conditions. A clear conclusion is that both the rate of ageing and the sensitivity of that rate to usage conditions, changes as the cell is used. In early stage ageing, Depth-of-Discharge is relatively insignificant, but in mid to late ageing shows a clear trend. Charge C-Rate appears to decrease in significance after the first month albeit remaining influential. 
It is also clear, that the relationship between usage conditions and capacity fade are not simple. There was evidence of higher order effects in both factors.

Depth-of-discharge and particularly Charge C-Rate appeared to gain significance above a certain threshold. There was also evidence of interaction effects between the two usage conditions.

Cycle time was shown to be, at the high temperature conditions tested, at least as influential as cycle throughput. It was shown that cycle time was a better indication of changes in the nature of cell ageing and was more consistent in the relationship between usage conditions and degradation than Ah throughput. This would be even truer in real conditions, which would likely have much larger time gaps between cycles, making time dominant in those cases.

The implications of this are that the modelling and control of ageing must account for the changing nature with cycle time, and the changing rate with usage conditions. It was shown however, that over a large amount of the cell lifetime, the capacity fade rate at high temperatures could be approximated as linear, when tracking the time at which the cells have been exposed to the high temperature conditions.

Last, it was shown that a lot of information can be gathered by applying a design-of-experiments approach to automotive cell ageing. To gain further insight however, techniques such as Incremental Capacity Analysis (ICA) should be combined with chemical analysis to gain a further insight into the underlying causes of ageing within the cell.

This will be explored in future work, along with an exploration of the nature of changes of resistance with ageing.

\section{ACKNOWLEDGEMENTS}

We would like to thank HORIBA MIRA and Coventry University for their support in this project.

\section{REFERENCES}

[1] European Commission, "Proposal for a Regulation of the European Parliament and of the Council: Setting emission performance standards for new passenger cars as part of the Community's integrated approach to reduce $\mathrm{CO} 2$ emissions from light-duty vehicles," 2018.

[2] European Commission, "A Roadmap for moving to a competitive low carbon economy in 2050," 2011.

[3] Ford Motor Company, "2016 Model Year Ford Hybrid Car and Electric Vehicle Warranty Guide," 2016.

[4] Renault UK Ltd, "Renault Z . E ./ Electric Vehicle Warranty terms and conditions including the $4+$ Warranty offer, applicable to all $\mathrm{Z}$. E . vehicles," 2016 .

[5] A. Hoke, A. Brissette, K. Smith, A. Pratt, and D. Maksimovic, "Accounting for Lithium-Ion Battery Degradation in Electric Vehicle Charging Optimization," IEEE J. Emerg. Sel. Top. Power Electron., vol. PP, no. 99, pp. 1-1, 2014.

[6] J. Vetter et al., "Ageing mechanisms in lithium-ion batteries," $J$. Power Sources, vol. 147, no. 1-2, pp. 269-281, 2005.

[7] A. Barré, B. Deguilhem, S. Grolleau, M. Gérard, F. Suard, and D. Riu, "A review on lithium-ion battery ageing mechanisms and estimations for automotive applications," J. Power Sources, vol. 241, pp. 680-689, 2013

[8] P. Keil, S. F. Schuster, P. Keil, S. F. Schuster, C. Von Lüders, and H. Hesse, "Lifetime Analyses of Lithium-Ion EV Batteries," in 3rd
Electromobility Challenging Issues conference (ECI), 2015, no. December.

[9] J. Remmlinger, S. Tippmann, M. Buchholz, and K. Dietmayer, "Lowtemperature charging of lithium-ion cells Part II: Model reduction and application," J. Power Sources, vol. 254, pp. 268-276, 2014.

[10] P. protocols for lithium-ion batteries and their impact on cycle life A. experimental study with different 18650 high-power cells Keil and A. Jossen, "Charging protocols for lithium-ion batteries and their impact on cycle life - An experimental study with different 18650 high-power cells," J. Energy Storage, vol. 6, pp. 125-141, 2016.

[11] R. Zhao, J. Liu, and J. Gu, "The effects of electrode thickness on the electrochemical and thermal characteristics of lithium ion battery," Appl. Energy, vol. 139, pp. 220-229, 2015.

[12] S. Panchal, I. Dincer, M. Agelin-chaab, R. Fraser, and M. Fowler, "Experimental and theoretical investigation of temperature distributions in a prismatic lithium-ion battery," Int. J. Therm. Sci., vol. 99, pp. 204-212, 2016.

[13] W. Prochazka, G. Pregartner, and M. Cifrain, "Design-of-Experiment and Statistical Modeling of a Large Scale Aging Experiment for Two Popular Lithium Ion Cell Chemistries," J. Electrochem. Soc., vol. 160, no. 8, 2013.

[14] L. Su et al., "Identifying main factors of capacity fading in lithium ion cells using orthogonal design of experiments," Appl. Energy, vol. 163, pp. 201-210, 2016

[15] Groot Jens, "Commercial xEV Battery Systems : Cycle Life Testing and Cell Modeling at AB Volvo," in Advanced Automotive Battery Conference Europe 2016, 2016.

[16] J. Groot, "State-of-Health Estimation of Li-ion Batteries : Cycle Life Test Methods," Chalmers University of Technology, 2012.

[17] T. Waldmann, M. Wilka, M. Kasper, M. Fleischhammer, and M. Wohlfahrt-Mehrens, "Temperature dependent ageing mechanisms in Lithium-ion batteries - A Post-Mortem study," J. Power Sources, vol. 262, pp. 129-135, 2014.

[18] T. M. Bandhauer, S. Garimella, and T. F. Fuller, "A Critical Review of Thermal Issues in Lithium-Ion Batteries," J. Electrochem. Soc., 2011.

[19] J. Groot, M. Swierczynski, A. Irina, and S. Knudsen, "On the complex ageing characteristics of high-power LiFePO 4 / graphite battery cells cycled with high charge and discharge currents," $J$. Power Sources, vol. 286, pp. 475-487, 2015.

[20] G. J. Offer, V. Yufit, D. A. Howey, B. Wu, and N. P. Brandon, "Module design and fault diagnosis in electric vehicle batteries," $J$. Power Sources, vol. 206, pp. 383-392, 2012.

[21] USABC, "Electric Vehicle Battery Test Procedures Manual Revision $2, " 1996$.

[22] S. F. Schuster et al., "Nonlinear aging characteristics of lithium-ion cells under different operational conditions," J. Energy Storage, vol. 1, no. 1, pp. 44-53, 2015.

[23] J. Jaguemont, L. Boulon, and Y. Dubé, “A comprehensive review of lithium-ion batteries used in hybrid and electric vehicles at cold temperatures," Appl. Energy, vol. 164, pp. 99-114, 2016.

[24] G. Ning, B. Haran, and B. N. Popov, "Capacity fade study of lithiumion batteries cycled at high discharge rates," J. Power Sources, vol. 117, no. 1-2, pp. 160-169, 2003.

[25] S. S. Choi and H. S. Lim, "Factors that affect cycle-life and possible degradation mechanisms of a Li-ion cell based on LiCoO2," J. Power Sources, vol. 111, no. 1, pp. 130-136, 2002.

[26] S. Watanabe, M. Kinoshita, T. Hosokawa, K. Morigaki, and K. Nakura, "Capacity fading of LiAlyNi1-x-yCoxO 2 cathode for lithium-ion batteries during accelerated calendar and cycle life tests (effect of depth of discharge in charge-discharge cycling on the suppression of the micro-crack generation of LiAlyNi 1-x-yCoxO2 parti,” J. Power Sources, vol. 260, pp. 50-56, 2014.

[27] R. . S. M Broussely, S Herreyre, P Biensan, P Kasztejna, K Nechev, "Aging mechanism in Li ion cells and calendar life predictions," $J$. Power Sources, vol. 97-98, pp. 13-21, 2001.

[28] M. Kassem, J. Bernard, R. Revel, S. Pélissier, F. Duclaud, and C. Delacourt, "Calendar aging of a graphite/LiFePO4 cell," J. Power Sources, vol. 208, pp. 296-305, 2012.

[29] S. Grolleau, A. Delaille, and H. Gualous, "Predicting lithium-ion battery degradation for efficient design and management," 2013 World Electr. Veh. Symp. Exhib., pp. 1-6, 2013. 
